\title{
Development of point-of-care testing devices to improve clozapine prescribing habits and patient outcomes
}

This article was published in the following Dove Press journal:

Neuropsychiatric Disease and Treatment

\author{
Shamir N Kalaria' \\ Deanna L Kelly ${ }^{2}$ \\ 'Center for Translational Medicine, \\ University of Maryland School of \\ Pharmacy, Baltimore, MD, USA; \\ ${ }^{2}$ Department of Psychiatry, School of \\ Medicine, Maryland Psychiatric Research \\ Center, University of Maryland School of \\ Medicine, Baltimore, MD, USA
}

\begin{abstract}
Although clozapine has demonstrated superior efficacy in patients with schizophrenia and other serious mental health illness, drug utilization rates are significantly low due to safety concerns and administration challenges. Previous research indicates that current barriers to clozapine use include lack of confidence and knowledge by prescriber, therapeutic monitoring requirements, lack of support and infrastructure to for adequate monitoring and patient adherence, and inadequate understanding of clozapine's benefit-risk profile by policy makers and payers. One potential solution to optimizing clozapine therapy and improving clinical outcomes is the use of point-of-care testing (POCT) devices. Although the drug development process for currently used therapeutics is widely acknowledged, little is known regarding the development of POCT devices by the clinical community. The aim of this review is to provide a summary of the regulatory approval process and current availability of POCT devices for monitoring clozapine therapeutics. The potential role of POCT devices in clinical trials to inform personalized dosing strategies and improve patient outcomes will also be discussed.
\end{abstract}

Keywords: clozapine, schizophrenia, patient outcomes, point-of-care testing devices, regulatory, therapeutic drug monitoring

\section{Introduction}

Schizophrenia is a severe and chronic psychiatric illness that affects approximately $1 \%$ of the world's population. ${ }^{1}$ The mainstay of treatment includes the use of antipsychotics that primarily mitigate positive symptoms associated with schizophrenia. Although many antipsychotic medications exist, their effectiveness has been reported to be similar. ${ }^{2}$ Clozapine was first approved in Europe in 1962, but it was not until 1975 when reports of agranulocytosis leading to death in clozapinetreated patients to voluntary market withdrawal. ${ }^{3,4}$ In late 1989, the US Food and Drug Administration (FDA) approved clozapine based on a trial that demonstrated effectiveness in patients with treatment-resistant schizophrenia (TRS). ${ }^{5}$ However, due to the increased risk of agranulocytosis, the FDA mandated, through the Clozaril Patient Management System that the distribution of clozapine be restricted to registered patients that are frequently monitored for hematological changes. Even though clozapine is the only antipsychotic approved for TRS, its underuse is well documented. ${ }^{6}$ Drug utilization rates for clozapine are considerably lower than the estimated prevalence of TRS. Current literature suggests that potential barriers for clozapine use include the complicated registration process, risk of adverse effects,
Correspondence: Deanna L Kelly Maryland Psychiatric Research Center (MPRC), 55 Wade Avenue, Catonsville 2I228, MD, USA

Tel + I 4I0402 686I

Email dlkelly@som.umaryland.edu 
need for hematologic monitoring, lack of prescriber education, and inadequate infrastructure to follow up patients. $^{7,8}$ Several generic formulations for clozapine are available on the market, and therefore medication costs are not a major barrier. In response to the stringent hematologic requirements for monitoring and the need to consolidate multiple patient registries, the FDA in 2015 proposed a Risk Evaluation and Mitigation Strategies (REMS) program to include a centralized registry, a lower absolute neutrophil count (ANC) threshold, recognition of patients with benign ethnic neutropenia (BEN) requiring an even lower threshold, and the removal of white blood cell count monitoring. ${ }^{6}$

A recent survey conducted by Kelly et al evaluated and confirmed potential barriers to clozapine use. Some of the most significant barriers were patient adherence to blood work and the burden of frequent monitoring (Figure 1). Another survey administered to practicing psychiatrists and residents suggested a potential solution to optimizing clozapine therapy and improving clinical outcomes should include monitoring clozapine levels and hematological parameters using point-ofcare devices. ${ }^{8}$ Routine therapeutic drug monitoring (TDM) has been widely accepted for medications that exhibit a narrow therapeutic window and when the majority of patients fail to achieve therapeutic targets after initial dosing. The goal of TDM with clozapine is to maximize treatment efficacy by alleviating symptoms associated with schizophrenia and minimize adverse effects. Current clozapine use guidelines recommend TDM in routine practice. ${ }^{9}$ However, inter- and intra-individual variability in clozapine drug clearance and lack of consensus on therapeutic targets makes optimizing therapy at the individual level challenging. ${ }^{10}$ Point-of-care testing (POCT) or "bedside testing" creates a decentralized method of rapidly obtaining clinical measures using smaller blood specimen to ultimately provide earlier results or alert clinicians to adjust their plan of action. POCT devices have consistently shown to improve patient outcomes while simultaneously decreasing health care costs. ${ }^{11,12}$ Considering additional benefits of clozapine such as reductions in hospitalization, aggression, and suicidality, the use of POCT may have a large impact in developing a more efficient TDM process. The aim of this review is to provide a summary regarding the United States (US) regulatory approval process and current availability of POCT devices for monitoring hematologic parameters and to measure clozapine concentrations. The role of POCT devices in clinical trials to inform personalized dosing strategies and improve patient outcomes will also be discussed.

\section{Approval process for POCT devices}

The FDA defines in-vitro diagnostics (IVDs) as

Reagents, instruments, and systems intended for use in diagnosis of disease or other conditions, including a determination of the state of health, in order to cure, mitigate, treat or prevent disease or its sequelae. Such [devices] are intended for use in the collection, preparation, and examination of specimens taken from the human body.

POCT devices are classified as IVDs that refers to the location where a test is performed, usually near the site of patient care. ${ }^{13}$ Administration of the device is commonly conducted by patients or personnel whose primary
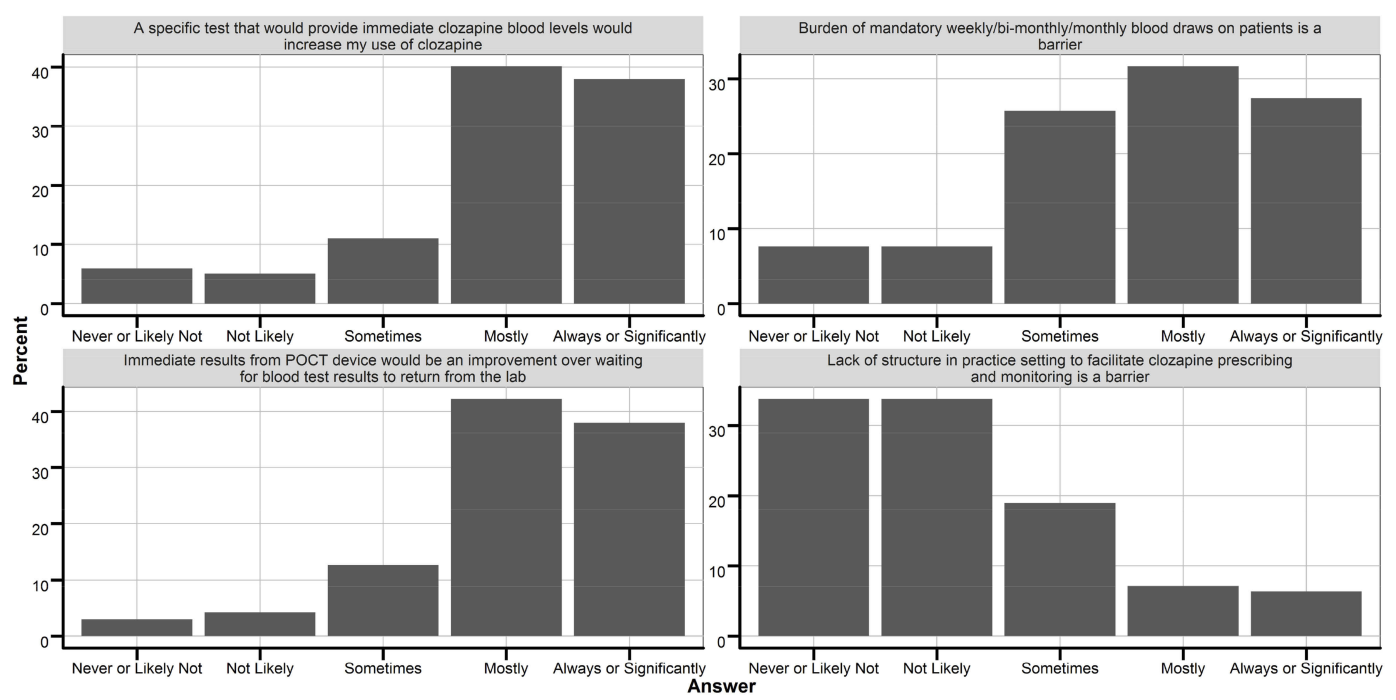

Figure I Responses to survey questions (Kelly et al, 20I8) regarding potential barriers and solutions to improving clozapine utilization. 
training is not in the clinical laboratory sciences. Regulatory oversight of IVDs is mandated by the FDA and/or by the Center for Medicaid and Medicare Services (CMS). ${ }^{14}$ The Clinical Laboratory Improvement Amendments (CLIA) were passed by US Congress and implemented by CMS to formally establish quality standards for laboratory testing to confirm appropriate accuracy, reliability, and efficient reporting of patient results regardless of the location of the actual testing procedure. ${ }^{15}$ IVDs are subject to both pre-market and post-market controls based on the FDA designated devices category and additionally subject to CLIA certification. In most cases, POCT devices are usually classified by the FDA as class II or III devices based on the intended use, indication, and level of risk posed to the patient or user. Under class II, devices are FDA-approved under a streamlined 510(k) pre-market notification process, while class III devices are approved under a pre-market approval procedure. ${ }^{13}$ With respect to the CLIA certification process, POCT devices can be classified as waived tests, tests of moderate complexity, or tests of high complexity. ${ }^{15,16}$ CLIA regulatory oversight can be waived if the test includes simple laboratory examinations and procedures that have been also cleared by the FDA for use at a patient's home, simple methodologies that have a "negligible probability of erroneous results, or tests with no reasonable risk of harm to a patient if performed incorrectly." Non-waived tests (moderate or high complexity tests) are required to satisfy specific criteria related to personnel use, quality management, operational and materials preparation, maintenance, and interpretation. ${ }^{17}$ POCT device developers are encouraged to apply for CLIA categorization during the FDA pre-market process.

Most POCT devices will fall under the moderate complexity CLIA classification category. According to $42 \mathrm{CFR}$ 493.15, waived tests are simple laboratory examinations which are cleared by the FDA for home or over-the-counter use. Manufacturers whose tests have been categorized as moderate complexity may request a waived classification by submitting a CLIA Waiver by Application to the FDA. Most recently, the FDA published a draft guidance outlining potential study designs for generating data to simultaneously support $510(\mathrm{k})$ and CLIA waiver pathways. Although this dual application approach is optional, it provides a more efficient method for manufacturers to market a new IVD device. ${ }^{18}$ Detailed descriptions of the content required in $510(\mathrm{k})$ and CLIA Waiver applications are provided in several FDA guidances. The FDA recommends manufacturers to provide information regarding the determination that a device is "simple," risk analysis, failure-alert/fail-safe mechanisms, usage variations under conditions of stress, analytical result, and results from comparison and reproducibility studies. ${ }^{18}$

\section{POCT devices to measure clozapine concentrations}

Measuring clozapine concentration levels can be used to evaluate medication adherence, therapeutic response, and safety risk in patients with schizophrenia. The ability to measure clozapine levels when initiating treatment can allow for optimized dosing regiments and improvement in patient outcomes. Since clozapine is metabolized by the hepatic cytochrome P540 enzymes (CYP1A2 and CYP3A4), drug interactions play a major role in influencing concentrations, especially in population that is vulnerable to polypharmacy. Smoking is also highly prevalent in the schizophrenia population. Patients that smoke exhibit lower drug concentrations than non-smokers. However, if a patient was to stop smoking, clozapine blood levels can be dangerously elevated to the reversal of CYP1A2 effects. ${ }^{19}$ Other factors such as acute infections can lead to high spikes in clozapine levels are also been a situation that warrants close serum level monitoring. ${ }^{20}$ Although target plasma levels of $250-350 \mathrm{ng} / \mathrm{mL}$ have been proposed for treating symptoms associated with schizophrenia, there is high variability in clinical response. Increasing concentrations $>350 \mathrm{ng} / \mathrm{mL}$ have also shown limited improvements in efficacy. ${ }^{20}$ Also, while this is variable, some have suggested that clozapine levels of $>1000 \mathrm{ng} / \mathrm{mL}$ may be associated with adverse effects and toxicity.

Currently, there are no available POCT devices that measure clozapine blood levels on the market. Ben-Yoav et al present an electrochemical lab on chip (LOC) sensing microsystem device that detects clozapine concentrations in real-time by utilizing a catechol-chitosan redox cycling system for signal amplification. ${ }^{21}$ The developed sensor is capable of detecting clozapine concentrations between 100 and $10,000 \mathrm{ng} / \mathrm{mL}$. In addition, this redox amplification method is also selective by differentiating clozapine from norclozapine. Even though traditional commercial benchtop methods demonstrate better selectivity and a lower limit of detection, the LOC microsystem could be a viable option as a POCT device to detect concentrations within the therapeutic range. ${ }^{21}$ This patented technology (US Patent Application Number: 15/034719) for detection is 
under development; however, the miniaturization and POCT platform for the device are not near market. Saladax has adapted immunoassay technology to detect clozapine and other antipsychotics with their MyCare Psychiatry line (pending FDA approval). The immunoassay for clozapine was found to be linear between $68 \mathrm{ng} /$ $\mathrm{mL}$ and $1500 \mathrm{ng} / \mathrm{mL}$. Within laboratory, precision was reported to be $<5.6 \%$. No endogenous or other drug products were found to interfere with the quantification of clozapine. When compared to the traditional liquid chromatography-tandem mass spectroscopy method, the developed immunoassay demonstrated a high degree of agreement with a correlation coefficient of 0.95 . In addition, Saladax is developing a small portable POCT innovation, utilizing the same immunoassay technology, to detect clozapine and other antipsychotic medications. ${ }^{22}$

\section{POCT devices to measure hematologic counts}

Severe neutropenia (ANC $<500 / \mu \mathrm{L}$ ) due to clozapine is well documented, leading to serious infections and even death in about $0.8 \%$ (or less) of patients treated. ${ }^{23}$ The safety risk does not appear to be dose dependent and is known to occur within the first 18 weeks of treatment. The updated REMS program has recently recognized BEN as a condition in certain ethnic groups who exhibit lower ANC counts on average as compared to standard laboratory ranges while not receiving treatment. Before initiating treatment, the FDA recommends that the baseline ANC must be at least $1500 / \mu \mathrm{L}$ for the general population and at least $1000 / \mu \mathrm{L}$ in patients diagnosed with $\mathrm{BEN}^{24}$ The required weekly ANC monitoring for 6 months places a large burden on patients, caregivers, and clinical staff. The obligatory monitoring of ANC has subsequently contributed to lower drug utilization rates and patient non-adherence. ${ }^{6}$

The HemoCue WBC Diff system is a POCT device that measures total white blood cell counts and differential counts (absolute and percentages of neutrophils, lymphocytes, monocytes, eosinophils and basophils) in capillary or venous whole blood using a finger prick method. The device is approved for marketing in Europe and still pending approval by the FDA (HemoCue WBC measures only white blood cell counts and is approved in the US). Approximately, $10 \mu \mathrm{L}$ of blood is needed to generate an accurate result. The reported measuring time is approximately 5 mins with a measurement range between 300 and $30,000 / \mu \mathrm{L}$. White blood cell counts were found to be linear across the measurement range with a correlation coefficient of 0.99 . In a study evaluating the performance of the device in clozapine users, the correlation between the POCT device and routine laboratory methods in measuring WBC and neutrophil counts in venous samples was 0.95. Correlation between capillary samples using the device and routine methods (venous sample) was 0.77 and 0.82 for WBCs and neutrophils, respectively. Intraassay reproducibility was consistent with manufacturer specifications for patients with low and high cell counts and when using capillary blood samples. ${ }^{25}$ In another study, a randomized cross-over trial design was used to compare conventional versus capillary blood sampling to assess the burden for patients and clinicians. A consistent trend was observed in favor of capillary blood testing as a significant number of patients reported less pain and anxiety, greater convenience, less fear, and a better understanding of the intended purpose of sample collection. Health care practitioners reported a greater advantage of using the POCT device that provided quick turnaround of analyzing the sample and reporting results. ${ }^{26}$

Chempaq XBC is another POCT device that is intended to measure hemoglobin, WBCs, and a 3-part differential (lymphocytes, monocytes, and neutrophils) using a similar finger stick method. The device is currently approved by the FDA and meets CLIA waiver requirements. Chempaq XBC utilizes impedance measurement to quantify WBC counts and differentials in a blood sample of $20 \mu \mathrm{L}$. Linearity was demonstrated between 1.4 and $100,000 / \mu \mathrm{L}$. Accuracy was determined using a predicate device (Coulter AT Diff Analyzer) which demonstrated a correlation of 0.99 when measuring WBC and neutrophils. Precision was found to be $<7 \%$ based on quality control WBC samples evaluated. In a study conducted by Rao et al, the Chempaq XBC device was compared with the laboratory Beckman Coulter analyzer to evaluate the performance of the POCT device. ${ }^{27}$ Precision, accuracy and linearity results were found to be within acceptable limits between the two methods. Comparison studies in various clinical settings such as the ER, primary care, ICU, and pediatric clinics demonstrated a high correlation $(r=0.97)$. A randomized cross-over trial also confirmed that patients favored monitoring using the capillary-based Chempaq XBC device as compared to traditional venous sampling methods. ${ }^{28}$

An alternative FDA-cleared POCT device is the Athelas One, which is indicated for the quantitative determination of WBC counts and neutrophil percentages using capillary or venous blood. Blood is collected into the Athelas test strips 
and the same is analyzed using computer vision technology. A smartphone or tablet is required to initiate the test and view test results. The Athelas device demonstrates a linear WBC measurement range of $1-20,000 / \mu \mathrm{L}$ and requires only $3.5 \mu \mathrm{L}$ of blood. A predicate device (Sysmex 500) was used in a comparison study which demonstrated $0 \%$ clinical range error and a high correlation between Athelas One and System 500 ( $\mathrm{r}=0.99$ and 0.97 for WBC counts and neutrophil percentages, respectively). Reproducibility/precision test results met predefined acceptance criteria. ${ }^{29,30}$ Results are returned within 5 mins and it has the capability of depositing ANC results automatically into the REMS system.

\section{Impact of POCT on precision therapeutics and patient outcomes}

Rapid point-of-care diagnostics are currently playing a major role in revolutionizing health care. ${ }^{31,32}$ The number of devices under development and approved by various regulatory agencies have increased over the last decade. ${ }^{33}$ Exposureresponse relationships for many antipsychotics vary greatly within and among patients. Several patient factors such a metabolizing status, smoking, food intake, and drug interaction could help explain the variability seen in drug concentrations. Although a clozapine concentration of $350 \mathrm{ng} / \mathrm{mL}$ and an ANC of $>1500 / \mu \mathrm{L}$ has been defined as an optimal target for efficacy and safety, these values are representative of an average subject. Limited knowledge is available linking drug exposures and clinical efficacy and safety outcomes at an individual level. It is possible that different subgroup patients may have inherently different therapeutic targets. Collection of data from POCT devices can assist in identifying different exposure targets to individualize therapy. ${ }^{34}$ Data from POCT services can be stored in repositories that can be leveraged to build quantitative relationships between concentrations and clinical outcomes. Based on previous surveys, there is a growing interest in providing continuous and structured care for patients initiated on clozapine therapy. Interdisciplinary, pharmacymanaged clinics that utilize POCT devices to monitor outpatient clozapine use can be instrumental. By providing an overview of the advances in POCT device development and their application to managing clozapine therapy, we hope to bridge these two communities to create newer technologies that will translate into meaningful outcomes.

\section{Disclosure}

Dr Deanna L Kelly reports honorarium for consultation from HLS Therapeutics and travel funding from Alkermes, outside the submitted work. In addition, Dr Kelly has a patent US9581536B2 issued and the author group has used the Athelas One device for a clinical trial from Athelas. The authors report no other conflicts of interest in this work.

\section{References}

1. McGrath J, Saha S, Chant D, Welham J. Schizophrenia: a concise overview of incidence, prevalence, and mortality. Epidemol Rev. 2008;30:67-76. doi:10.1093/epirev/mxn001

2. Leucht S, Cipriani A, Spineli L, et al. Comparative efficacy and tolerability of 15 antipsychotic drugs in schizophrenia: a multipletreatment s meta-analysis. Lancet. 2013;382:951-962. doi:10.1016/ S0140-6736(13)60733-3

3. Crilly J. The history of clozapine and its emergence in the US market: a review and analysis. Hist Psychiatry. 2007;18:39-60. doi:10.1177/ 0957154X07070335

4. Nucifora FC, Mihaljevic M, Lee BJ, Sawa A. Clozapine as a model for antipsychotic development. Neurotherapeutics. 2017;14:750-761. doi:10.1007/s13311-017-0552-9

5. Kane J, Honigfeld G, Singer J, et al. Cloazpaine for the treatmentresistant schizophrenic: a double blind comparison with chlorpromazine. Arch Gen Psychiatry. 1988;45:789-796. doi:10.1001/ archpsyc.1988.01800330013001

6. Curry B, Palmer E, Mounce C, et al. Assessing prescribing practices of clozapine before and after the implementation of an updated risk evaluation and mitigation strategy. Ment Health Clin. 2018;8:63-67. doi:10.9740/mhc.2018.03.063

7. Sayer M, Love R, Freudenreich O, et al. M115: Improving clozapine use in the united states: a survey of barriers and solutions informing a workgroup to devise a national strategy. Schizophr Bull. 2017;43: S252. doi:10.1093/schbul/sbx022.110

8. Kelly DL, Ben-Yoav H, Payne GF, et al. Blood draw barriers for treatment with clozapine and development of a point-of-care monitoring device. Clin Schizophr Relat Psychoses. 2018;12:23-30. doi:10.3371/CSRP.KEBE.070415

9. Himeke C, Bauman P, Bergemann N, et al. AGNP consensus guidelines for therapeutic drug monitoring in psychiatry: update 2011. Pharmacopsychiatry. 2011;44:195-235. doi:10.1055/s-0031-1286287

10. Remington G, Agid O, Foussais G, et al. Clozapine and therapeutic drug monitoring: is there sufficient evidence for an upper threshold? Psychopharmacology. 2013;225:505-518. doi:10.1007/s00213-0122922-7

11. St John A. The evidence to support point of care testing. Clin Biochem Rev. 2010;31:111-119.

12. Price CP, Kricka LJ, Kricka LJ. Improving healthcare accessibility through point-of-care technologies. Clin Chem. 2007;53:1665-1675. doi:10.1373/clinchem.2006.084707

13. US Department of Health and Human Services. Protection of human subjects. 21 CFR 809.3; Revised April 1, 2018. Available from: https://www.accessdata.fda.gov/scripts/cdrh/cfdocs/cfcfr/CFRSearch. $\mathrm{cfm}$ ?FR=809.3 Accessed December 6, 2018.

14. Van Norma GA. Drugs, devices, and the FDA: part 2: an overview of approval processes: FDA approval of medical devices. JACC Basic Transl Sci. 2016;1:277-287. doi:10.1016/j.jacbts.2016.03.009

15. Center for Medicare and Medicaid Services (CMS). Department of Health and Human Services: Clinical Laboratory Improvement Amendments of 1988 (CLIA): 1998.

16. US Food and Drug Administration (FDA) Guidance for industry and FDA staff. Administrative procedures for CLIA categorization. 2017.

17. US Food and Drug Administration (FDA) Guidance for industry and FDA staff. Select updates for recommendations for clinical laboratory improvement amendments of 1988 (CLIA) Waiver Applications for manufacturers of in-vitro diagnostic devices. 2018. 
18. US Food and Drug Administration (FDA) Guidance for industry and FDA staff. Recommendations for dual $510(\mathrm{k})$ and CLIA waiver by application studies. 2018.

19. Goossen RB, Freeman DJ, Satchell AM, Urquhart BL. Monitoring clozapine: are fingerprick blood and plasma clozapine levels equivalent to arm venipuncture blood and plasma levels? Ther Drug Monit. 2003;25:469-472.

20. Ellison JC, Dufresne RL. A review of the clinical utility of serum clozapine and norclozapine levels. Ment Health Clin. 2015;5:68-73. doi:10.9740/mhc.2015.03.068

21. Ben-Yoav H, Chocron SE, Winkler TE, et al. An electrochemical microsystem for clozapine antipsychotic treatment monitoring. Electrochim Acta. 2015;163:260-270. doi:10.1016/j.electacta.2015.02.112

22. Salamone S, Courtney JB, Sard H; Inventors. Saladax Biomedical Inc. Clozapine immunoassay. US patent 8,771,972 B2. 2011 July 8.

23. Siskand D, McCartney L, Goldschlager R, et al. Clozapine vs. firstand second generation antipsychotics in treatment refractory schizophrenia: a systematic review and meta-analysis. $\mathrm{Br} J$ Psychiatry. 2016;209:385-392. doi:10.1192/bjp.bp.115.177261

24. U.S. Food and Drug and Administration. FDA modifies monitoring for neutropenia associated with schizophrenia medicine clozapine; approves new shared REMS program for all clozapine medicines. 2015.

25. Bui HN, Bogers JP, Cohen D, Njo T, Herruer MH. Evaluation of the performance of a point-of-care method for total and differential white blood cell count in clozapine users. Int J Lab Hem . 2016;38:703-709. doi:10.1111/ijlh.2016.38.issue-6

26. Bogers JP, Bui H, Herruer M, Cohen D. Capillary compared to venous blood sampling in clozapine treatment: patients' and healthcare practitioners' experiences with a point-of-care device. Eur Neuropsychopharmacol. 2015;25:319-324. doi:10.1016/j.euroneuro. 2014.11.022
27. Rao LV, Ekberg BA, Connor D, et al. Evaluation of a new point of care automated complete blood count (CBC) analyzer in various clinical settings. Clin Chim Acta. 2008;389:120-125. doi:10.1016/j. cca.2007.12.006

28. Nielsen J, Thode D, Stenager E, et al. Hematological clozapine monitoring with a point-of-care device: a randomized cross-over trial. Eur Neuropsychopharmacol. 2012;22:401-405. doi:10.1016/j. euroneuro.2011.10.001

29. Athelas. Clinical third party accuracy validation of the Athleas device: CBC device compared with venous Sysmex 5000 in POC clinic. Published August1, 2017. Available from: https://athelas.com/ a4ab6c781c91ee7290ced2991f4036e9.pdf. Accessed December 1, 2018.

30. US Food and Drug Administration (FDA). Guidance for industry and FDA staff. Class II special controls guidance document: premarket notifications for automated differential cell counter for immature or abnormal blood cells. 2001.

31. Rooney KD, Schilling UM. Point of care testing in the overcrowded emergency department-can it make a difference? Crit Care. 2014;18:692-699. doi:10.1186/s13054-014-0692-9

32. St John A, Price CP. Economic evidence and point-of-care testing. Clin Biochem Rev. 2013;34:61-74.

33. Wang P, Kricka LJ. Current and emerging trends in point-of-care technology and strategies for clinical validation and implementation. Clin Chem. 2018;64:10-14. doi:10.1373/clinchem.2017. 282970

34. Guest PC, Martins-de-Souza D. Enabling point-of-care testing and personalized medicine for schizophrenia. NPJ Schizophr. 2017;3:1. doi:10.1038/s41537-016-0005-1
Neuropsychiatric Disease and Treatment

\section{Publish your work in this journal}

Neuropsychiatric Disease and Treatment is an international, peerreviewed journal of clinical therapeutics and pharmacology focusing on concise rapid reporting of clinical or pre-clinical studies on a range of neuropsychiatric and neurological disorders. This journal is indexed on PubMed Central, the 'PsycINFO' database and CAS, and

\section{Dovepress}

is the official journal of The International Neuropsychiatric Association (INA). The manuscript management system is completely online and includes a very quick and fair peer-review system, which is all easy to use. Visit http://www.dovepress.com/testimonials.php to read real quotes from published authors. 